\title{
Co-encapsulation of Rifampicin and Isoniazid in Crude Soybean Lecithin Liposomes
}

\author{
Christian I. Nkanga ${ }^{a} \mathbb{D}^{\S}$, Xavier S. Noundou ${ }^{a} \mathbb{D}^{\mathbb{D}}$, Roderick B. Walker ${ }^{\mathrm{b}}$ and Rui W.M. Krause ${ }^{\mathrm{a}, \star}$ (D) \\ ${ }^{a}$ Centre for Chemico- and Bio-Medicinal Research (CCBR), Department of Chemistry, Faculty of Science, Rhodes University, \\ P.O. Box 94, Grahamstown, 6140, South Africa. \\ ${ }^{b}$ Division of Pharmaceutics, Faculty of Pharmacy, Rhodes University, P.O. Box 94, Grahamstown, 6140, South Africa.
}

Received 23 September 2018, revised 2 April 2019, accepted 3 April 2019.

\begin{abstract}
Despite the well-known anti-mycobacterial actions of isoniazid (INH) and rifampicin (RIF), the clinical success of tuberculosis (TB) therapy requires prolonged administration of multiple drugs in high doses, which often result in frequent adverse effects and low patient adherence. Although liposomes are promising candidates for controlled delivery of anti-TB drug, the high cost of synthetic and highly purified natural lipids currently used in liposomal technology might preclude the universal application of therapeutic liposomes. This work aimed at evaluating the potential of a cost-effective lipid material, crude soybean lecithin (CL), to co-encapsulate RIF and INH for liposomal dual delivery. RIF was encapsulated in CL-liposomes with/without cholesterol using film hydration method, after which INH was incorporated using a freeze-thawing technique. Dynamic light scattering, differential scanning calorimetry, $X$-ray diffraction and dialysis were used for liposome characterization. Liposomes containing CL alone (CLL) exhibited $90 \%$ encapsulation efficiency for RIF and $59 \%$ for INH. The mean size and surface charge of CLL were $1114 \mathrm{~nm}$ and $-63 \mathrm{mV}$, respectively. In addition, CLL showed a controlled release profile for the co-encapsulated drugs. CLL would be promising vehicles for macrophage-targeting drug delivery. The present findings demonstrate the feasibility of using CL for preparation of combination products for liposomal delivery.
\end{abstract}

KEYWORDS

Tuberculosis, isoniazid, rifampicin, drug delivery, liposomes, soybean lecithin.

\section{Introduction}

Tuberculosis (TB) is a microbial infection that is currently part of the most dominating public health concerns. ${ }^{1}$ Despite the significant decrease in incidence from 2004 to $2014,{ }^{2}$ TB remains a common deadly infectious disease in developing countries, where one TB-infected patient dies every 15-20 seconds. ${ }^{1,3,4}$ Rifampicin (RIF) and isoniazid (INH) are two drugs used in the current regimen for TB therapy and, with pyrazinamide (PYZ) and ethambuthol (ETM), form part of first-line anti-TB drug therapy. ${ }^{4,5}$ There is evidence that RIF can inhibit DNAdependent RNA polymerase of intra-macrophage microorganisms, such as Mycobacterium tuberculosis. ${ }^{6,7}$ The sterilizing effect of INH may be explained by its ability of inhibiting synthesis of mycolic acid and producing active free radicals inside the mycobacterial organism..$^{8-10}$ Although each of these medicines is effective, their use in combination for TB therapy is recommended as monotherapy often results in resistance. ${ }^{1,11,12}$ RIF associated with INH exhibits high cure rates of up to 95-98\% when treating susceptible organisms. However, hepatic first pass metabolism and nonselective bio-distribution of these drugs may result in a significant decrease in bioavailability when using currently available dosage forms. As a result, clinical success of TB therapy requires prolonged administration of multiple drugs in high doses, which often cause side effects that result in poor patient adherence to the treatment. ${ }^{5,13}$ These issues justify an urgent need to develop new strategies to improve anti-TB therapy.

Considering the biological localization of M. tuberculosis, mostly within macrophages, ${ }^{14}$ the use of particulate drug carriers that

* To whom correspondence should be addressed. E-mail: r.krause@ ru.ac.za can be taken up by cells of the mononuclear phagocytic system (CMPS) represents a promising alternative therapeutic approach. The work by Parikh et al. is a typical illustration of the potential of colloidal vehicles to achieve controlled anti-TB drug delivery to the CMPS. Using INH-loaded polymeric microparticles (of poly- $\varepsilon$-caprolactone), the authors observed much higher drug accumulation inside alveolar macrophages than non-encapsulated INH administered via the same route (i.e. inhalation as a dry powder). ${ }^{15}$ Among drug vehicles that can afford macrophage passive targeting (based on their colloidal nature), liposomes represent the most commonly investigated due to their first ranking as the most clinically established drug carriers. ${ }^{16}$ Liposomes are phospholipid-based vesicular systems that are increasingly being investigated for antimicrobial drug delivery. ${ }^{17}$ Apart from their well-known ability to be avidly engulfed by the CMPS, ${ }^{18,19}$ liposomes hold several advantages that justify their clinical reputation and therapeutic success. The advantages of liposomes over other drug vehicles include high biocompatibility and biodegradability, low toxicity and poor immunogenicity, improved drug solubility and controlled distribution. ${ }^{16,19}$ Moreover, liposomes offer the advantage of easy surface modification, which adds on the possibility for active targeting, on top of the native passive targeting feature. ${ }^{14,20}$ The macrophage active targeting properties can be obtained by decorating liposomes surface with negative charges or specific receptor ligands. An extensive illustration of the macrophagetargeting concepts could be the work by Vyas et al. ${ }^{21}$ In comparison with non-decorated/neutral liposomes, this research group observed enhanced intramacrophage accumulation of the $O$-steroyl amylopectin-decorated and negatively charged 
liposomes. Kaul et al. ${ }^{22}$ also witnessed the active targeting concept, when studying folate-receptor targeted liposomes for non-invasive co-delivery of RIF and ofloxacin to macrophages in inflammatory lesions. This recent example also illustrates another unique feature of liposomal vehicles, viz. the ability to accommodate multiple cargoes, which aligns well with the recommended multidrug therapy for TB and has been investigated for co-encapsulation of first-line anti-TB drugs. ${ }^{23}$

There are reports discussing the potential of liposomes to co-encapsulate INH and RIF for dual delivery. Deol et al..$^{24}$ have developed a liposomal system composed of egg-yolk phosphatidylcholine, cholesterol, distearoylphosphatidylethanolamine-polyethyleneglycol 2000, dicetylphosphate and $\mathrm{O}$-stearyl amylopectin for the delivery of INH and RIF. These liposomes demonstrated a sterilizing effect in M. tuberculosisinfected mice following intravenous administration of two doses of formulation per week for six weeks. Labana et al. ${ }^{25}$ studied pharmacokinetic parameters such as the maximum plasmatic concentration (Cmax), the area under the curve (AUC) and the elimination rate constant $(\mathrm{Kel})$ following weekly intravenous administration of the same formulation. In general, the liposomes exhibited greater accumulation of the two drugs in the lungs, spleen and liver in addition to better outcomes in infected animals when compared to the free drugs. Another illustration could be the work reported by Gürsoy et al. ${ }^{26}$ These authors used egg-yolk phosphatidyl-choline type XI-E and dipalmitoyl-phosphatidyl-choline in combination with cholesterol (Chol) for liposomal co-encapsulation of INH and RIF. These liposome formulations demonstrated sustained release of the compounds and were deemed to be promising vehicles for improving the pharmacokinetics of therapeutic molecules.

Despite the potentials of liposomes as a delivery system, the high cost of liposomal technology, because of the need for expensive synthetic or high-grade natural phospholipids, ${ }^{27,28}$ might preclude its universal application. The economic status of formulation materials is particularly relevant when addressing poverty related diseases like TB. Therefore, we have previously set ourselves an ambitious goal of looking into potential strategies towards development of affordable liposomal technologies for widespread application of therapeutic liposomes in the developing world. As a start, crude soybean lecithin (which is a cheap FDA-approved natural lipid mixture $)^{29}$ was evaluated as a cost-effective option for liposomal encapsulation of small hydrophilic drug molecules like INH. The liposomes formulated with crude soybean lecithin exhibited much higher encapsulation efficiency $(79 \%)$ than those made of purified soybean lecithin (21\%). ${ }^{29}$ However, since these liposomes showed bust release profiles, our group has further performed hydrazone derivatization of INH prior to liposomal encapsulation. This led to a soybean lecithin liposome-based formulation that demonstrated remarkable $\mathrm{pH}$-dependent release characteristics. ${ }^{30}$ Thereafter, as this concept showed some success, hydrazone conjugation of INH to a phthalocyanine (as a fluorescent tag) was considered for multimodal system development, combining $\mathrm{pH}$-triggered drug release with the possibility for bioimaging assessments of crude soybean lecithin liposomes. ${ }^{31}$ Although these liposomebased systems look promising for advanced delivery studies, their applicability in TB biological systems may be limited by the issue of antimicrobial resistance, because of which the use of single drugs is not recommended. ${ }^{1}$

Hence, the present work aimed at assessing the potential of crude soybean lecithin to co-encapsulate INH and RIF for liposomal dual delivery. Various crude soybean lecithin liposomes were formulated and evaluated in comparison with liposomes composed of purified soybean lecithin. This study demonstrated the feasibility of using crude soybean lecithin for preparation of liposome-based combination products.

\section{Materials and Methods}

\subsection{Materials}

Soybean lecithin (granules) was purchased from Health Connection Wholefoods (USA). The supplier indicated the following composition for $100 \mathrm{~g}$ of the granules: phosphatidylcholines (23 g), phosphatidylinositols (14 g), polyunsaturated fats (35 g), saturated fats $(13 \mathrm{~g})$, glycaemic carbohydrates $(8 \mathrm{~g})$, sodium $(0.11 \mathrm{~g})$ and about $2940 \mathrm{~kJ}$ of total energy. Isoniazid (INH), rifampicin (RIF), tetraethylammonium bromide (T-Eth.Br) mono- and dibasic sodium phosphates $\left(\mathrm{NaH}_{2} \mathrm{PO}_{4}\right.$ and $\mathrm{Na}_{2} \mathrm{HPO}_{4}$ ) were from Sigma Aldrich (Germany). Methanol of HPLC grade was purchased from Merck (Germany) and acetonitrile from Ranbaxy Fine Chemicals Ltd (India). Acetone and ethyl acetate were purchased from Protea Chemicals (South Africa). Chloroform was purchased from BM Scientific (South Africa). A Rotavapor R-205 (Büchi, Switzerland) rotary evaporator and Allegra 64R (Beckman Coulter, USA) centrifuge were used to produce and harvest the liposomes. A Digital Ultrasonic Cleaner (Spellbound 909, UK) was used for sonication and a Lyo Lab 3000 lyophilizer (Apollo Scientific CC, South Africa) was used for freeze-drying products. Water used in this study was HPLC grade (ultrapure) 18 mega ohm water, prepared using a Milli-Q academic A10 water purification system (Millipore ${ }^{\circledR}$, Bedford, MA, USA) consisting of an Ion- $X^{\circledast}$ ion-exchange cartridge and a quantum EX-ultrapore Organex ${ }^{\circledR}$ cartridge fitted with a $0.22 \mu \mathrm{m}$ Millipak ${ }^{\circledR}$ stack filter (Millipore ${ }^{\circledR}$, Bedford, MA, USA).

\subsection{Purification of Crude Lecithin}

For comparative purposes, purified lecithin was produced from crude soybean lecithin as previously described by Mertins et al..$^{32}$ Briefly, $10 \mathrm{~g}$ of the granules of crude soybean lecithin was dispersed in ethyl acetate $(100 \mathrm{~mL})$ and ultrapure water $(4 \mathrm{~mL})$ was added under gentle shaking, leading to formation of a biphasic system. After decantation, the supernatant was discarded, and the lower phase was dispersed in acetone $(60 \mathrm{~mL})$. This led to formation of agglomerates that were disrupted by means of a glass rod prior to removal of washing acetone. A fresh portion of acetone was added to further disrupt the agglomerates. The resultant precipitate was vacuum filtered and allowed to dry in a desiccator at room temperature for $48 \mathrm{~h}$. The prepared purified lecithin $(7.99 \mathrm{~g})$ was stored at room temperature.

\subsection{Preparation of Dual Liposomes}

The liposomes were prepared in two stages in order to encapsulate both RIF and INH. The formulation compositions evaluated are summarized in Table 1. A thin film hydration method was used for liposome preparation. The procedures used for subsequent loading of the two drugs are detailed in the following subsections.

\subsubsection{Preparation of RIF-loaded Liposomes}

Following the details presented in Table 1, the liposomes formulations CL-F1, CL-F2 and CL-F3 were prepared using different amounts of cholesterol in the compositions (0-50\% of total lipid, $200 \mathrm{mg}$ ), while the other formulations were prepared without incorporating or investigating cholesterol. The following paragraphs detail the sequential approach used for preparation of RIF-liposomes in the presence and absence of cholesterol. 
Table 1 Description of the composition of liposomal formulations.

\begin{tabular}{ccccc}
\hline Formulation code & Lecithin type & Lecithin-Chol mass ratio & Lipid-RIF mass ratio & Lipid-INH mass ratio \\
\hline CL-F1 & Crude & $1: 0$ & $1: 0.25$ & $1: 2$ \\
CL-F2 & Crude & $1: 0.33$ & $1: 0.25$ & $1: 2$ \\
CL-F3 & Crude & $1: 1$ & $1: 0.25$ & $1: 2$ \\
CL-F4 & Crude & $1: 0$ & $1: 0.25$ & $1: 0$ \\
CL-F5 & Crude & $1: 0$ & $1: 0$ & $1: 0$ \\
CL-F6 & Crude & $1: 0$ & $1: 0$ & $1: 2$ \\
PL-F1 & Purified & $1: 0$ & $1: 0.25$ & $1: 0$ \\
PL-F2 & Purified & $1: 0$ & $1: 0$ & $1: 2$ \\
PL-F3 & Purified & $1: 0$ & & \\
\hline
\end{tabular}

2.3.1.1. Preparation of RIF-liposomes in the Presence of Cholesterol

For each formulation, lecithin and cholesterol were separately weighed and put together to yield $200 \mathrm{mg}$ of total lipid (according to the composition presented in Table 1). This lipid mixture was placed in a $25 \mathrm{~mL}$ round-bottom flask containing $50 \mathrm{mg}$ of RIF and $2 \mathrm{~mL}$ of chloroform was added for dissolution. The organic solvent was removed by means of a rotary evaporator set at $60{ }^{\circ} \mathrm{C}$ for $5 \mathrm{~min}$. The whole flask was placed in a desiccator under vacuum overnight at room temperature. Ultrapure water $(6 \mathrm{~mL})$ was added to the dried thin film and hydration was undertaken at $60{ }^{\circ} \mathrm{C}$ for $60 \mathrm{~min}$ under stirring at $400 \mathrm{rpm}$. The suspension obtained was red and was transferred to a $50 \mathrm{~mL}$ centrifuge tube, while rinsing with Ultrapure water $(3 \mathrm{~mL})$ to yield centrifugation volume level. The preparation was centrifuged for $5 \mathrm{~min}$ at $4000 \mathrm{rpm}$ to facilitate sedimentation of free RIF as hydrophobic particles. ${ }^{33}$ The supernatant containing RIFloaded liposomes was decanted and retained for further processing (i.e. incorporation of INH).

\subsubsection{Preparation of RIF-liposomes in the Absence of Cholesterol}

Lecithin $(200 \mathrm{mg})$ and chloroform $(2 \mathrm{~mL})$ were sequentially added to RIF (50 mg) placed in a $25 \mathrm{~mL}$ round-bottom flask. Upon complete dissolution, the organic solvent was removed from the resultant solution by means of a rotary evaporator for thin film formation. The resultant film was hydrated, and the preparation was processed as described in Section 2.3.1.1. The isolated RIF-loaded liposomes suspension was retained for further incorporation of INH. All RIF-free formulations were prepared under the same conditions. Three different batches were prepared for each formulation.

\subsubsection{Incorporation of Isoniazid}

The supernatant containing the liposomes was transferred to a Beckman centrifuge tube containing $400 \mathrm{mg}$ INH and formulated as compositions listed in Table 1 . The mixture was gently hand-shaken and warmed for $3 \mathrm{~min}$ at $40^{\circ} \mathrm{C}$ in order to increase the rate of dissolution of INH. The tube was subjected to a bath sonication over $20 \mathrm{~min}$ at $60{ }^{\circ} \mathrm{C}$. The preparation was then subjected to two freeze-thaw cycles. Each cycle included freezing for $5 \mathrm{~min}$ in liquid nitrogen, followed by thawing for $10 \mathrm{~min}$ at $60{ }^{\circ} \mathrm{C} .^{34}$ The liposomes were equilibrated for $60 \mathrm{~min}$ at room temperature prior to addition of ultrapure water $(20 \mathrm{~mL})$ for effective centrifugation of the preparation. The centrifugation of the mixture was undertaken for $20 \mathrm{~min}$ at $20000 \mathrm{rpm}$ and $20^{\circ} \mathrm{C}$. The supernatant was decanted and retained for estimation of the encapsulation efficiency for INH. The residual liposomes (pellet) were rinsed five times with ultrapure water $(20 \mathrm{~mL})$ and freeze-dried further. The solid liposomes produced were stored away from light at room temperature prior to further evaluations. Any INH-free liposomes were prepared as described.

\subsection{Characterization of Drug-loaded Liposomes}

\subsubsection{Evaluation of Encapsulation Efficiency (EE)}

\subsubsection{EE for RIF}

HPLC grade methanol ( $30 \mathrm{~mL}$ ) was added to the RIF particles isolated following centrifugation and diluted $1 / 10$ prior to analysis using the HPLC method developed by Mariappan et al..$^{35}$ and modified as described herein. To calculate the EE, a preparation free of lipid components (RIF-control solution) was prepared following the same procedure used to manufacture liposomes. Quantitation of this solution was used to establish a theoretical total concentration of RIF ( $\mathrm{tC})$. The concentration of un-encapsulated RIF was established (uC). The percentage EE (\% EE) was calculated using Equation 1:34

$$
\% \mathrm{EE}=\frac{\mathrm{tC}-\mathrm{uC}}{\mathrm{tC}} \times 100
$$

\subsubsection{EE for INH}

The supernatant isolated during INH incorporation was diluted 1/10 using HPLC grade methanol. A control solution of INH was prepared using the same conditions and commences by dissolving INH (400 mg) in the lipid-free RIF-control solution in order to estimate the theoretical total concentration $(\mathrm{tC})$ of INH. The concentration of un-encapsulated INH in the supernatant $(\mathrm{uC})$ was used to calculate the EE. Samples were analyzed using a slightly modified HPLC method developed by Mariappan et al..$^{35}$ for the simultaneous analysis of INH and RIF.

The HPLC system used was an Agilent HP1100 LC-MSD (GMI Company, USA) equipped with a quaternary solvent delivery module, in-line degasser, DAD detector, 1100 MSD and ChemStation software was used for the collection and analysis of chromatographic data. Separation was achieved using a Zorbax Elipse Plus C18 4.6 i.d. $\times 150 \mathrm{~mm} \times 5 \mu \mathrm{m}$ stationary phase for reversed-phase HPLC analysis and gradient elution. The mobile phase consisted of methanol (solvent A) and monobasic sodium phosphate $0.01 \mathrm{M}$ containing $0.05 \% \mathrm{w} / \mathrm{v}$ T-Eth.Br adjusted to $\mathrm{pH} 3.5$ using orthophosphoric acid (solvent B). The column was equilibrated for $15 \mathrm{~min}$ with $90 \% \mathrm{v} / \mathrm{v}$ solvent $\mathrm{B}$ and $10 \% \mathrm{v} / \mathrm{v}$ solvent $\mathrm{A}$. Two minutes following the first sample injection, the amount of solvent $B$ in the mobile phase was decreased proportionally by $3 \%$ per minute over $15 \mathrm{~min}$. Then, the proportion of solvent A was increased at a rate of $1 \%$ per minute for $40 \mathrm{~min}$. Before starting a new experiment, the composition of the mobile phase was allowed for equilibrium over $15 \mathrm{~min}$. The retention time of INH and RIF was $1.6 \pm 0.2 \mathrm{~min}$ and $29.9 \pm 1.1 \mathrm{~min}$, respectively. The \% EE of INH in liposomes was calculated using Equation 1 and the results indicate that formulation CL-F1 was suitable for further characterization. All the EE experiments were conducted in triplicate. 


\subsubsection{Determination of Particle Size and Zeta Potential}

The measurements of particle size, polydispersity index and Zeta potential were performed at room temperature on a Zetasizer ZEN-3600 MAL1043132 (Malvern Instruments, UK) set at the scattering angle of $173^{\circ}$. Freeze-dried liposomes were ubjected to redispersion in ultrapure water prior to dynamic light scattering (DLS) experiments. The sample was loaded in either an ordinary or capillary cuvette $d$ for particles size or Zeta potential determination, respectively.

\subsubsection{Particle Shape}

The particle shape analysis was done by microscopic observation using a Transmission Electron Microscope, TEM (Carl Zeiss, USA). An aliquot of liposomes suspension prepared in Section 2.4.2. was deposited on a copper grid drop wise and liquid in excess was adsorbed filter paper. The sample was air-dried for $48 \mathrm{~h}$ prior to microscopic visualization.

\subsubsection{Differential Scanning Calorimetry (DSC)}

DSC experiments were conducted to investigate the polymorphism of the liposomes in conjunction with the free drugs. A DSC-6000 instrument (Perkinelmer, USA) was used along with the software DSC Pyris series for data processing. All the samples were heated at $30-445^{\circ} \mathrm{C}$ (with $10^{\circ} \mathrm{C} \mathrm{min}^{-1}$ as flow rate). The atmosphere of the sample chamber was saturated with

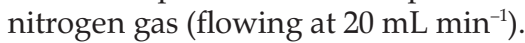

\subsection{5. $X$-ray Diffraction (XRD)}

The crystalline nature of the drugs co-encapsulated was assessed by XRD. The XRD patterns were recorded on an XRD D8 Discover instrument (Bruker, USA) equipped with a Lynx Eye detector, using a nickel filter and $\mathrm{Cu}-\mathrm{K} \alpha$ radiation set at 1.5404 angstrom. The samples were loaded onto a glass-based sample holder and the scans were run at 2-theta range 10-60 , using a slit width and step size of $6.0 \mathrm{~mm}$ and $1^{\circ} \mathrm{min}^{-1}$, respectively.

\subsubsection{Drug Release Study}

The dual liposome formulation that showed the highest EE (CL-F1) was used for in vitro release studies in addition to formulations that contained cholesterol (CL-F2 and CL-F3) and solutions of free drug for comparative purposes. The content and release of INH and RIF were studied using the procedures that had been reported by Rojanarat et $a l . .^{36}$ and Chimote and Banerjee $^{37}$, respectively, with slight modifications as described below.

Fifty $\mathrm{mg}$ of freeze-dried sample was dispersed in phosphate buffer (pH 7.4) in a $10 \mathrm{~mL}$ volumetric flask, and $1 \mathrm{~mL}$ of the resul- tant suspension was diluted 1/10 with HPLC grade methanol. The mixture was sonicated for $30 \mathrm{~min}$ at $35^{\circ} \mathrm{C}$ to promote liposomes disruption and facilitate dissolution of INH and RIF. The resulting solution was filtered five times with a $0.45 \mu \mathrm{m}$ syringe filter and the HPLC analysis undertaken as described. Quantitation was achieved by interpolation using freshly prepared calibration curves. The INH and RIF content (\%DC) was estimated using Equation 2:

$$
\% \mathrm{DC}=\frac{\text { Amount of drug determined }}{\text { Total mount of the preparation }} \times 100
$$

A $1 \mathrm{~mL}$ aliquot of the liposome dispersion was transferred to a piece of dialysis bag (Membra-Cel MD 10-14 × 100 CLR, Sigma Aldrich, Germany) that was placed in a glass vial containing $20 \mathrm{~mL}$ buffer ( $\mathrm{pH}$ 7.4) and a magnetic stirrer. This receptor medium was maintained at $37^{\circ} \mathrm{C}$ whilst stirring at $240 \mathrm{rpm} .{ }^{38}$ At $0.5,1,1.5,2,3,4,5,7,9$ and $12 \mathrm{~h}, 5 \mathrm{~mL}$ of the release medium was withdrawn for HPLC analysis. The aliquot sampled was replaced by the same volume of the fresh buffer ( $\mathrm{pH}$ 7.4) to maintain sink condition. Three replicate experiments were performed for each drug release study.

\section{Results and Discussion}

\subsection{Encapsulation Efficiency}

The EE of the crude lecithin liposomes for INH and RIF was found to decrease with the increase in cholesterol content (as shown in Table 2). The same phenomenon was previously observed when RIF was encapsulated alone using phosphatidyl choline from soybean lecithin. ${ }^{39}$ This could be due to potential saturation of the liposomal membranes when RIF and cholesterol are used simultaneously, as both compounds are lipophilic and tend to preferentially locate in lipid bilayers. ${ }^{18,40}$ This saturation may also have affected INH loading during the freeze-thawing process, which relies on diffusion of INH molecules to into liposomal vesicles. ${ }^{34}$

Surprisingly, the liposomes manufactured using crude lecithin alone (CL-F1) exhibited the highest EE for INH and RIF, $59.03 \pm$ $0.68 \%$ and $90.10 \pm 1.15 \%$, respectively. These data are promising as results of a previous study reported an EE of 8-10\% for INH and $44-49 \%$ for RIF. $^{24}$ Gürsoy et al. $^{26}$ reported an EE of $73.84 \pm$ $0.78 \%$ for INH and $81.53 \pm 2.06 \%$ for RIF. However, the lipid to drug mass ratios used in this study were 1:0.027 for INH and 1:0.000027 for RIF, whereas for CL-F1 INH and RIF were co-encapsulated using lipid to drug mass ratios of $1: 2$ and 1:0.25, respectively.

In addition, all liposome batches manufactured using purified lecithin exhibited much lower EE than those made of crude lecithin (Table 2). Regardless of the type of lecithin used, the

Table 2 Characteristics of the liposome formulations $(n=3)$.

\begin{tabular}{|c|c|c|c|c|c|}
\hline \multirow[t]{2}{*}{ Formulation code } & \multirow[t]{2}{*}{$\begin{array}{c}\mathrm{PS} \pm \mathrm{S} . \mathrm{D} . \\
/ \mathrm{nm}\end{array}$} & \multirow[t]{2}{*}{ PDI \pm S.D. } & \multirow[t]{2}{*}{$\begin{array}{c}\mathrm{ZP} \pm \mathrm{S} . \mathrm{D} . \\
/ \mathrm{mV}\end{array}$} & \multicolumn{2}{|c|}{$\begin{array}{c}\mathrm{EE} \pm \mathrm{S} . \mathrm{D} . \\
1 \%\end{array}$} \\
\hline & & & & $\mathrm{INH}$ & RIF \\
\hline CL-F1 & $1114.00 \pm 42.37$ & $0.34 \pm 0.06$ & $-63.30 \pm 2.13$ & $59.03 \pm 0.68$ & $90.10 \pm 1.15$ \\
\hline CL-F2 & $894.10 \pm 18.30$ & $0.44 \pm 0.03$ & $-50.80 \pm 1.48$ & $57.13 \pm 2.03$ & $64.93 \pm 4.51$ \\
\hline CL-F3 & $1514.00 \pm 132.40$ & $0.36 \pm 0.11$ & $-48.04 \pm 0.93$ & $45.65 \pm 1.52$ & $55.24 \pm 2.80$ \\
\hline CL-F4 & $594.10 \pm 5.88$ & $0.35 \pm 0.09$ & $-59.60 \pm 2.10$ & * & $66.33 \pm 0.15$ \\
\hline CL-F5 & $568.00 \pm 8.89$ & $0.35 \pm 0.18$ & $-20.30 \pm 0.71$ & $53.21 \pm 1.33$ & * \\
\hline CL-F6 & $546.80 \pm 0.46$ & $0.54 \pm 0.04$ & $-57.90 \pm 2.68$ & $*$ & * \\
\hline PL-F1 & $758.60 \pm 3.61$ & $0.43 \pm 0.04$ & $-45.50 \pm 3.39$ & $43.91 \pm 0.59$ & $78.69 \pm 1.63$ \\
\hline PL-F2 & $543.50 \pm 14.13$ & $0.61 \pm 0.02$ & $-56.00 \pm 1.08$ & $*$ & $59.99 \pm 0.13$ \\
\hline PL-F3 & $687.80 \pm 87.07$ & $0.71 \pm 0.19$ & $-35.60 \pm 4.80$ & $34.65 \pm 2.43$ & * \\
\hline
\end{tabular}


co-loaded liposomes showed significantly higher EE than liposomes containing a single compound $(P$-value $<0.05)$. This observation agrees with the findings published by Gürsoy et al. ${ }^{26}$ and suggests the existence of an advantageous interaction between INH and RIF, which might have promoted simultaneous loading into liposomes. However, it was observed that the \%EE values of INH in both mono- and dually-loaded liposomes (CL-F5 and CL-F1) were below the value previously obtained with crude soybean lecithin (about $79 \%) .{ }^{29}$ Since the same freeze-thawing method was used for INH loading, the difference in $\% \mathrm{EE}$ could be due to the presence of cholesterol in the previous INH-loaded liposomes (unlike in CL-F5). Nevertheless, the \% EE of CL-F1 for INH appears to be of great interest when considering the previous findings reported elsewhere; ${ }^{36}$ with INH \% EE of about $18-30 \%$, and $36.7 \pm 1.8 \% .{ }^{37}$ Same for RIF, its \%EE in CL-F1 $(90.10 \%)$ was found to be greater than the values reported by other groups; such as Patil et al. $(79.25 \%)^{39}$ and Manca et al. $(76 \%) \cdot{ }^{13}$ Considering the impact of the entrapment capacity on the cost of the drug carriers, ${ }^{41}$ high \%EE demonstrated by CL-F1 may add more economical values to crude soybean lecithin for potential development of costeffective liposomal technology. ${ }^{29}$

\subsection{Particle Size and Zeta Potential}

The liposome formulation CL-F1 has exhibited a mean particles size of $1114.00 \pm 42.37 \mathrm{~nm}$, which falls within the range that has been deemed suitable for effective uptake by alveolar macrophages, 1000-3000 nm. ${ }^{42}$ Irrespective of the type of lecithin, INH- and RIF-loaded liposomes were significantly larger than the empty and single drug-loaded liposomes (Table 2).

No significant differences for Zeta potential were observed when comparing empty and drug-loaded liposomes $(P$-value $>$ 0.05). However, formulations containing INH alone exhibited a lower surface charge regardless the type of lecithin used (Table 2). The change in Zeta potential may be due to the presence of INH molecules in the polar regions of the vesicle membranes. ${ }^{40}$ Formulation CL-F1 exhibited a highly negative surface charge, $-63.30 \pm 2.13 \mathrm{mV}$, which is potentially useful for prolonged stability of these liposomes, as charged particles are

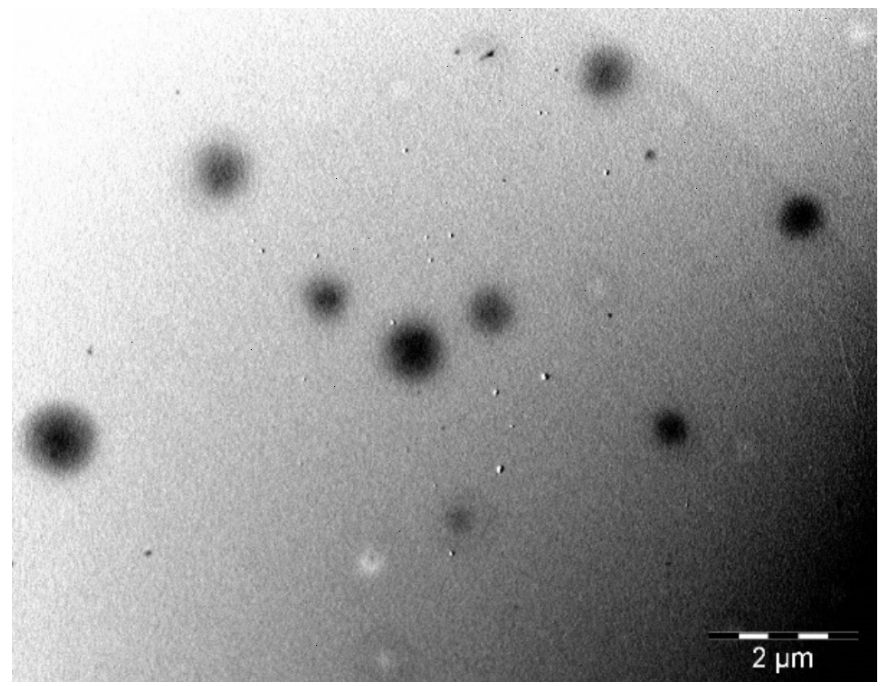

Figure 1 Typical TEM micrograph of the liposomes CL-F1.

likely to be dispersed as individual particles due to the repulsive forces. ${ }^{19}$ In addition, this negative particle charge may be promising for the biological fate of CL-F1 liposomes, considering the fact that macrophage scavenger receptors recognize negatively charged particles ${ }^{43}$ and $M$. tuberculosis mostly reside inside macrophages. ${ }^{14}$

\subsection{Particle Shape}

As shown in Fig. 1, the formulation CL-F1 exhibited the presence of individual particles that were spherical in shape and characteristic of liposomal vesicles. The absence of aggregated liposomes suggests the presence of repulsive forces due to the density of surface charges between liposomes. This observation also supports the data from particle size determination done by DLS, which is often limited by particle aggregation. ${ }^{19}$

\subsection{DSC}

The endothermic peaks observed in the DSC curves of the pure drugs (Fig. 2A and Fig. 2F) were shifted to lower values in CL-F1 (Fig. 2C). In the heat flow curve of CL-F1 the peak at

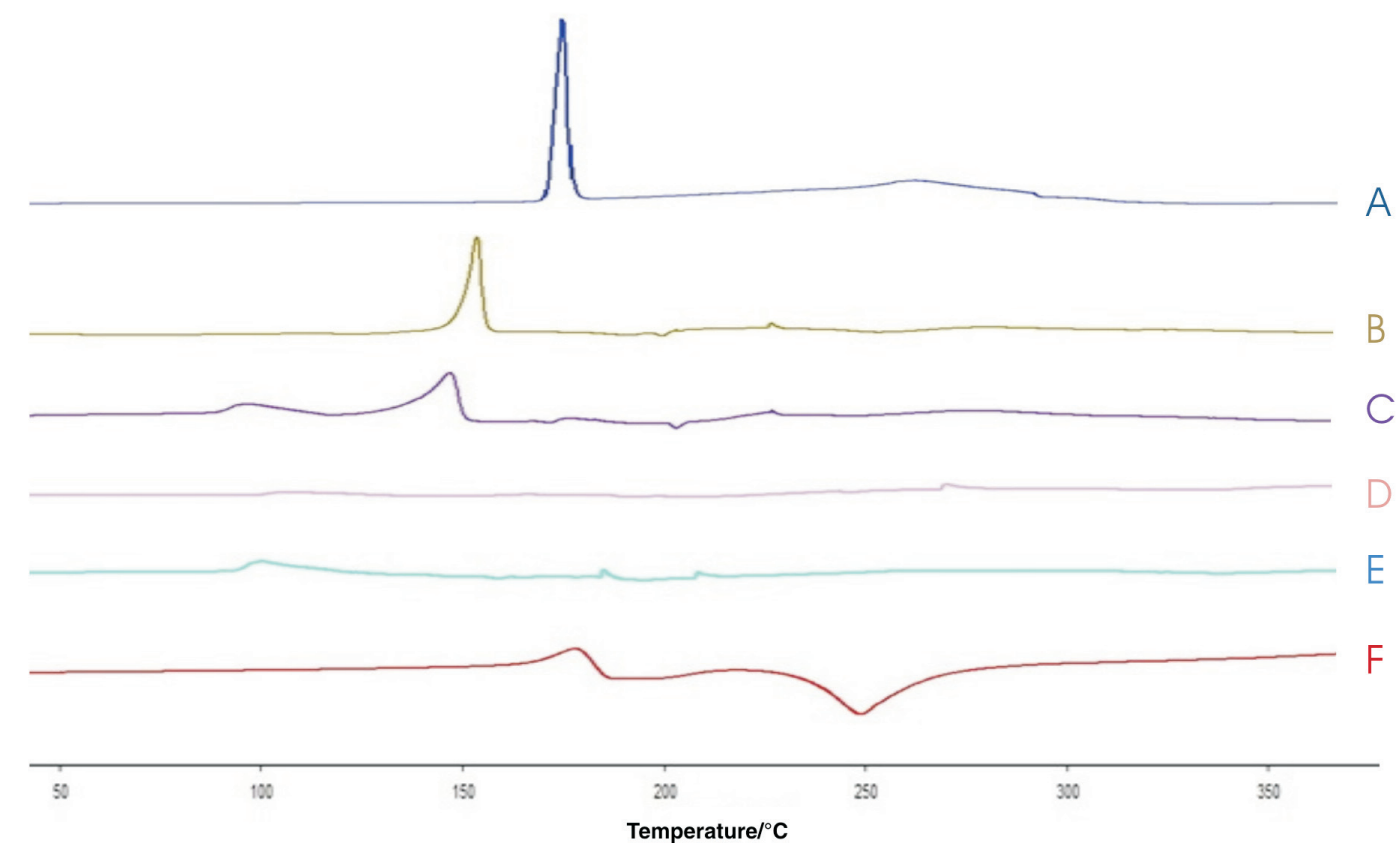

Figure 2 DSC thermograms of pure INH (A); CL-F5 (B); CL-F1 \{co-loaded\} (C); CL-F6 (D); CL-F4 (E) and RIF (F). 


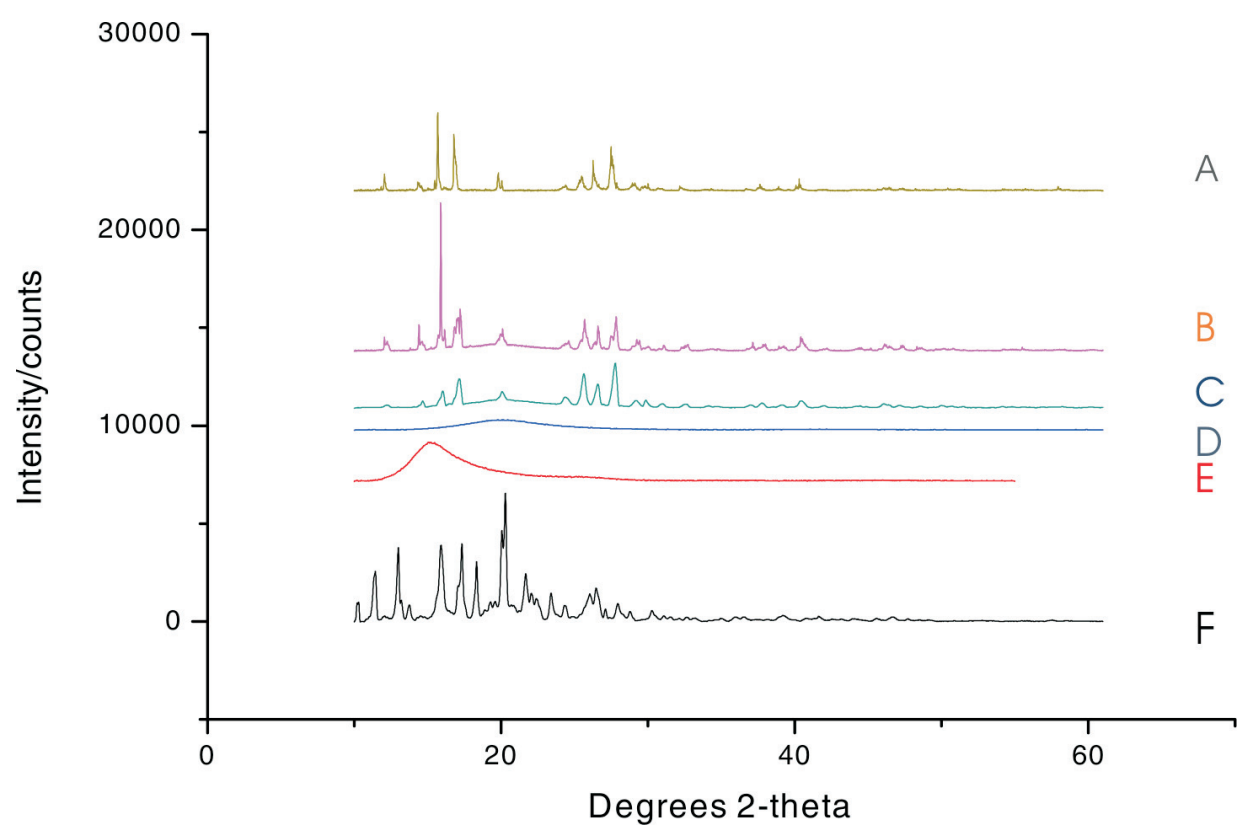

Figure 3 XRD patterns of pure INH (A); CL-F5 (B); CL-F1 \{co-loaded\} (C); CL-F6 (D); CL-F4 (E) and RIF (F).

$121.29^{\circ} \mathrm{C}$ can be allocated to RIF and the one at $165.71^{\circ} \mathrm{C}$ to INH, considering the DSC profiles of the mono-drug-loaded liposomes (CL-F4 and CL-F5) and empty liposomes (CL-F6). Comparing CL-F1 to the mono-drug-loaded liposomes, the melting point of RIF seems to decrease significantly ( $P$-value $<$ $0.05)$. This is consistent with the observation reported elsewhere and suggests that RIF is getting amorphous in liposomes. ${ }^{39,44}$ The probable explanation should be the fact that RIF's molecules are deeply embedded into the lipid bilayers as suggested by Rodrigues et al. ${ }^{40}$

\subsection{XRD}

CL-F1 showed major crystalline peaks at positions 15-18, 20 and $25-30^{\circ}$ (Fig. 3C). These might be related to the presence of INH that has also exhibited almost the same diffraction profile as pure drug (Fig. 3A), since pure RIF showed some peaks only at 17-20 and $20-25^{\circ}$ (Fig. 3F) that are not seen either in CL-F1 or CL-F4 (Fig. 3E). While the X-RD profile of CL-F5 is almost like that of CL-F1, CL-F4 shows the same halo pattern with the empty liposomal formulation (CL-F12). This confirms the loss of crystallinity of RIF in liposomes revealed by DSC and previously reported in the literature. ${ }^{39,44}$

\subsection{Drug Release Profile}

The release of INH was more rapid than that of RIF as free drug or from the liposome formulations. This may be due to INH being more water soluble than RIF as it has an aqueous solubility of $125 \mathrm{mg}$ per $\mathrm{mL}$ when compared to $1-2 \mathrm{mg}$ per $\mathrm{mL}$ for RIF. ${ }^{5}$ The release rate for INH and RIF from CL-F1 liposomes appears to be slower than that of each free drug (Fig. 4).

Patil et al. ${ }^{39}$ observed a decrease in the rate of RIF release as cholesterol content increased, whereas in these studies no major difference was observed in the kinetics of RIF release for the liposome formulations tested (Fig. 5). However, the release of INH appears to be affected by the presence of cholesterol; with $85 \%, 81 \%$ and $72 \%$ INH respectively released from batches

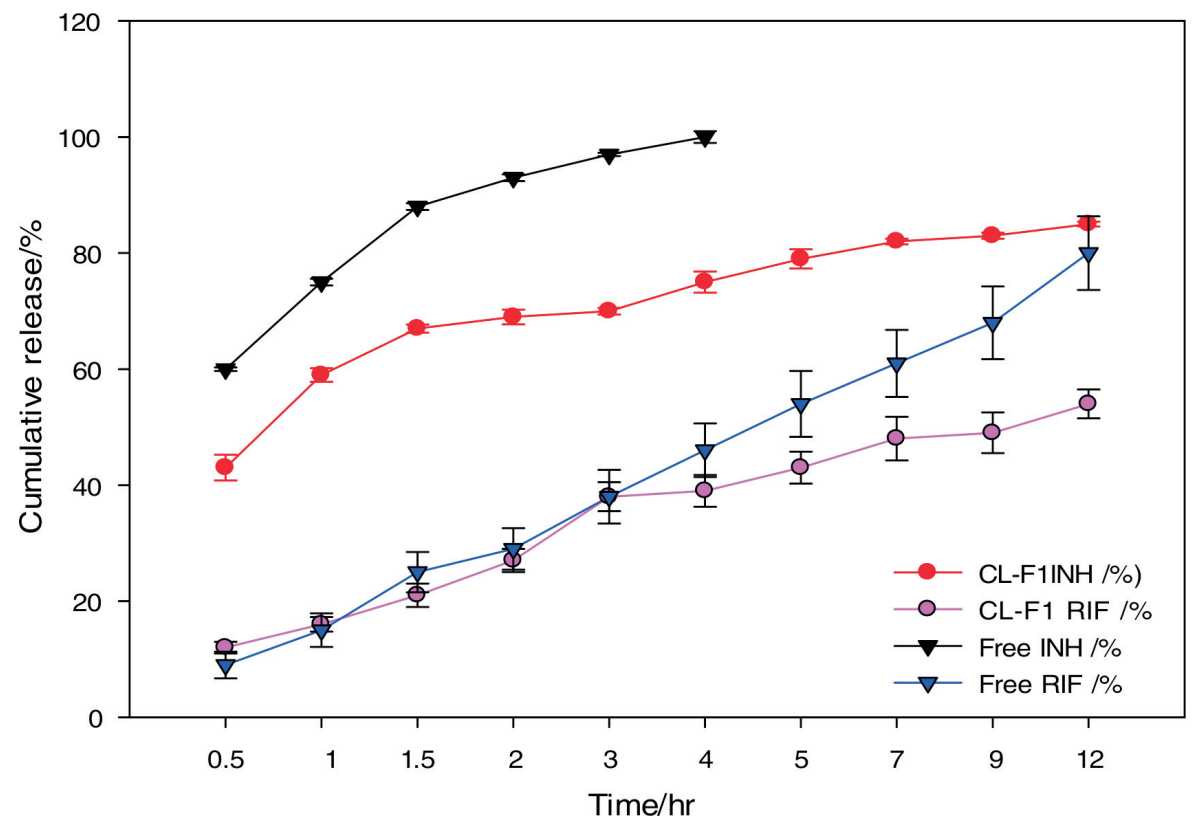

Figure 4 Comparison of INH and RIF release from liposomes (CL-F1) and free drug $(n=3)$. 


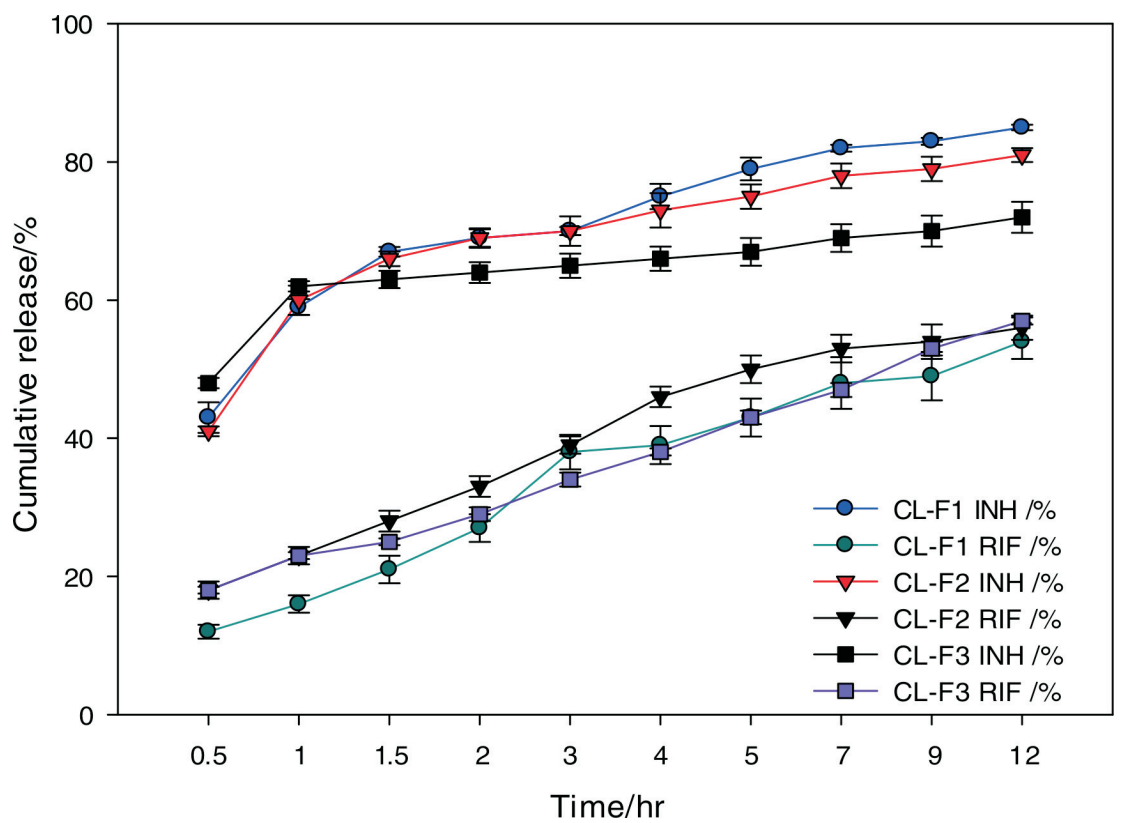

Figure 5 Comparison of release profiles from batches CL-F1, CL-F2 and CL-F3 $(n=3)$.

CL-F1, CL-F2 and CL-F3 over $12 \mathrm{~h}$. This result is probably due to a reduction in membrane permeability as cholesterol content increases the packing density of the phospholipid bilayers. ${ }^{18,19}$ Although the dual release observed from CL-F1 liposomes was slower than the dissolution of free drugs, there was a large difference in the percentage INH and RIF released from these liposomes over $12 \mathrm{~h}, 85 \%$ and $56 \%$ release for each compound, respectively. Consequently, the batch CL-F1 is a promising formulation for dual delivery of INH and RIF in terms of pharmacological outcomes, as INH is used for rapid activity against $M$. tuberculosis whereas RIF ensures more permanent bactericidal activity albeit being released at a slower rate. ${ }^{12}$

\section{Conclusion}

The liposomes prepared using crude soybean lecithin (CL) alone in this study have successfully co-encapsulated INH and RIF more efficiently than those prepared using cholesterol and/or purified soybean lecithin. The mean particles size and Zeta potential of dual carrier CL-based liposomes suggest that macrophage-targeting following pulmonary delivery is possible from literature-based evidence. ${ }^{18,19,42-45}$ In addition, CL-based liposome exhibited controlled release profiles that may be useful for enhancing the pharmacological outcomes of RIF and INH. This delivery feature could enhance anti-TB drugs bioavailability and reduce medication requirements for treating TB. Further pharmacological evaluations and in vivo testing of this technology are required to define the value of the dual carrier liposomes, specifically in terms of antimicrobial activity, cytotoxicity, pulmonary deposition, macrophage uptake and pharmacokinetic evaluation of the encapsulated drugs. To the best of our knowledge, there is no report that discusses the potential of crude soybean lecithin to co-encapsulate RIF and INH for liposomal dual delivery. This study proposes crude soy bean lecithin as an alternative to synthetic or highly purified natural lipids for preparation of liposomal combination products. These results suggest that investigations on liposomal delivery of other existing and emerging drugs using readily available lipid mixtures, such as crude rice lecithin, egg lecithin and others; may result in the development of affordable liposomal technologies.

\section{Acknowledgements}

The authors acknowledge the financial supports provided by the NGO Förderverein Uni Kinshasa e. V., fUNIKIN, ElseKroner-Fresenius Stiftung and Holger-Poehlmann Foundation through the excellence scholarship programme 'Bourse d'Excellence Bringmann aux Universités Congolaises, BEBUC', and the Sandisa Imbewu Programme of Rhodes University. Funding from the National Research Foundation (NRF) is also acknowledged.

\section{Declaration of interest}

None.

\section{${ }^{s}$ ORCID iDs}

C.I. Nkanga

X.S. Noundou

R.W.M. Krause

orcid.org/0000-0001-7712-478X

orcid.org/0000-0002-8667-8351 orcid.org/0000-0001-6788-6449

\section{References}

1 World Health Organization, WHO Report: Global tuberculosis report, Geneva, Switzerland, 2017.

2 P. Kielstra and T. Zoe, eds., Ancient enemy, modern imperative - A time for greater action against tuberculosis. Economist Insights. The Economist Group, 2014.

Online at: http://www.janssen-emea.com/sites/default/files/TB\%20 report_WEB.pdf

3 V. Dartois, The path of anti-tuberculosis drugs: from blood to lesions to mycobacterial cells, Nat. Rev. Microbiol., 2014, 12(3), 159-167.

4 P. Piccini, E. Chiappini, T. Enrico, M. de Martino and L. Galli, Clinical peculiarities of tuberculosis, BMC Infect. Dis., 2014, 14 (Suppl.1), 1-12.

5 A. Sosnik, M.Á. Carcaboso, J.R. Glisoni, A.M. Moretton and A.D. Chiappetta, New old challenges in tuberculosis: potentially effective nanotechnologies in drug delivery, Adv. Drug Deliv. Rev., 2010, 62, $547-559$.

6 A. Feklistov, V. Mekler, Q. Jiang, L.F. Westblade, H. Irschik, R. Jansen, A. Mustaev, S.A. Darst and R.H. Ebright, Rifamycins do not function by allosteric modulation of binding of $\mathrm{Mg}^{2+}$ to the RNA polymerase active center, Proc. Natl. Acad. Sci. USA, 2008, 105 (39), 14820-14825.

7 E.A. Campbell, N. Korzheva, A. Mustaev, K. Murakami, S. Nair, A. Goldfarb and S.A. Darst. Structural mechanism for rifampicin inhibition of bacterial RNA polymerase. Cell, 2001, 104 (6), 901-912.

8 Z. Ahmad, L.G. Klinkenberg, M.L. Pinn, M.M. Fraig, C.A. Peloquin, W.R. Bishai, E.L. Nuermberger, J.H. Grosset and P.C. Karakousis, Biphasic kill curve of isoniazid reveals the presence of drug-tolerant, 
not drug-resistant, Mycobacterium tuberculosis in the Guinea pig, J. Infect. Dis., 2009, 200 (7), 1136-1143.

9 J. Suarez, K. Ranguelova, A.A. Jarzecki, J. Manzerova, V. Krymov, X. Zhao, S. Yu, L. Metlitsky, G.J. Gerfen and R.S. Magliozzo, An oxyferrous heme/protein-based radical intermediate is catalytically competent in the catalase reaction of Mycobacterium tuberculosis catalaseperoxidase (KatG), J. Biol. Chem., 2009, 284 (11), 7017-7029.

10 B. Lei, W. Chih-Jen and T. Shiao-Chun, Action mechanism of antitubercular isoniazid: activation by Mycobacterium tuberculosis KatG, isolation, and characterization of InhA inhibitor, J. Biol. Chem., 2000, 275 (4), 2520-2526.

11 C.M. Yuen, A.W. Tolman, T. Cohen, J.B. Parr, S. Keshavjee and M.C. Becerra, Isoniazid-resistant tuberculosis in children: a systematic review, Pediatr. Infect. Dis. J., 2013, 32 (5), e217-e226.

12 N. Kumar, P. Kumar, K. Pramod, M. Kumar and R. Kumar, Nanotechnology: a focus on treatment of tuberculosis. Review, Int. J. Drug Deliv., 2011, 3, 25-42.

13 M.L. Manca, C. Sinico, M.A. Maccioni, O. Diez, A.M. Fadda and M. Manconi, Composition influence on pulmonary delivery of rifampicin liposomes, Pharmaceutics, 2012, 4, 590-606.

14 A.F. Tăbăran and C. Catoi, Macrophages targeted drug delivery as a key therapy in infectious disease, Biotechnol.Mol. Biol. Nanomed., 2014, 2, 2330-9326.

15 R. Parikh, D. Sonali, A. Pooja and P. Leena, Inhaled microparticles of antitubercular antibiotic for in vitro and in vivo alveolar macrophage targeting and activation of phagocytosis, J. Antibiot., 2014, 67, 387-394.

16 U. Bulbake, D. Sindhu, K. Nagavendra and K. Wahid, Liposomal formulations in clinical use: an updated review, Pharmaceutics 2017, 9, 12 DOI: 10.3390/pharmaceutics9020012

17 L. Zhang, D. Pornpattananangku, C.M. Hu and C.M. Huang, Development of nanoparticles for antimicrobial drug delivery, Curr. Med. Chem., 2010, 17, 585-594.

18 G. Bozzuto and A. Molinari, Liposomes as nanomedical devices, Int. J. Nanomedicine, 2015, 10, 975-999.

19 B.S. Pattni, V.V. Chupin and V.P. Torchilin, New developments in liposomal drug delivery, Chem. Rev., 2015, 115, 10938-10966.

20 B. Patel, N. Gupta and F. Ahsan, Particle engineering to enhance or lessen particle uptake by alveolar macrophages and to influence the therapeutic outcome, Eur. J. Pharm. Biopharm., 2015, 89, 163-174.

21 S.P. Vyas, M.E. Kannan, S. Jain, V. Mishra and P. Singh, Design of liposomal aerosols for improved delivery of rifampicin to alveolar macrophages, Int. J. Pharm., 2004, 269, 37-49.

22 A. Kaul, S. Chaturvedi, A. Attri, M. Kalra and A.K. Mishra, Targeted theranostic liposomes: rifampicin and ofloxacin loaded pegylated liposomes for theranostic application in mycobacterial infections, RSC Adv., 2016, 6, 28919-28926.

23 P. Walvekar, R. Gannimani and T. Govender, Combination drug therapy via nanocarriers against infectious diseases, Eur. J. Pharm. Sci., 2019, 127, 121-141.

24 P. Deol, G.K. Khuller and K. Joshi, Therapeutic efficacies of isoniazid and rifampicin encapsulated in lung-specific stealth liposomes against Mycobacterium tuberculosis infections induced in mice, Antimicrob. Agents Chemother., 1997, 41, 6, 1211-1214.

25 S. Labana, R. Pandey, S. Sharma and G.K. Khuller, Chemotherapeutic activity against murine tuberculosis of once weekly administered drugs (isoniazid and rifampicin) encapsulated in liposomes, Int. J. Antimicrob. Agents, 2002, 20, 301-304.

26 A. Gürsoy, K. Ece and Ö. Sumru, Co-encapsulation of isoniazid and rifampicin in liposomes and characterization of liposomes by derivative spectroscopy, Int. J. Pharm., 2004, 271, 115-123.
27 J. Li, X. Wang, Z. Ting, W. Chunling, H. Zhenjun, L. Xiang and D. Yihui, A review on phospholipids and their main applications in drug delivery systems, Asian. J. Pharma. Sci., 2015, 10, 81-98.

28 D. Yokota, M. Moraes and S.C. Pinho, Characterization of lyophilized liposomes produced with non-purified soy lecithin: a case study of casein hydrolysate microencapsulation, Braz. J. Chem. Eng., 2012, 29 (2), 325-335.

29 C.I. Nkanga, R.W. Krause, X.S. Noundou and R.B. Walker, Preparation and characterization of isoniazid-loaded crude soybean lecithin liposomes, Int. J. Pharm., 2017, 526 (1), 466-473.

30 C.I. Nkanga, R.B. Walker and R.W. Krause, pH-Dependent release of isoniazid from isonicotinic acid (4-hydroxy-benzylidene)-hydrazide loaded liposomes, J. Drug. Deliv. Sci. Technol., 2018, 45, 264-271.

31 C.I. Nkanga and R.W. Krause. Conjugation of isoniazid to a zinc phthalocyanine via hydrazone linkage for $\mathrm{pH}$-dependent liposomal controlled release, Appl. Nanosci., 2018, 8(6), 1313-1323.

32 O. Mertins, M. Sebben, P.H. Schneider, A.R. Pohlmann and N.P. Silveira, Caracterização da pureza de fosfatidilcolina da soja através de RMN de 1 H e de 31P, Quim. Nova, 2008, 31, 1856-1859.

33 G. Patel, M. Chougule, M. Singh and M. Ambikanandan, Nanoliposomal dry powder formulations, Methods Enzymol., 2009, 464, 167-191.

34 A.P. Costa, X. Xu and D.J. Burgess, Freeze-anneal-thaw cycling of unilamellar liposomes: effect on encapsulation efficiency, Pharm. Res., 2014, 31, 97-103.

35 T.T. Mariappan, B. Singh and S. Singh, A validated reversed-phase (C18) HPLC method for simultaneous determination of rifampicin, isoniazid and pyrazinamide in USP dissolution medium and simulated gastric fluid, Pharm. Pharmacol. Commun., 2000, 6, 345-349.

36 W. Rojanarat, N. Changsan, E. Tawithong, S. Pinsuwan, H.K. Chan and T. Srichana, Isoniazid proliposome powders for inhalationpreparation, characterization and cell culture studies, Int. J. Mol. Sci., 2011, 12, 4414-4434.

37 G. Chimote and R. Banerjee, In vitro evaluation of inhalable isoniazid-loaded surfactant liposomes as an adjunct therapy in pulmonary tuberculosis, J. Biomed. Mater Res. (Part B): Appl. Biomater., 2010, 94B, $1-10$.

38 R.O. Cook, R.K. Pannu and I.W. Kellaway, Novel sustained release microspheres for pulmonary drug delivery, J. Control. Release, 2005, 104, 79-90.

39 J.S. Patil, V.K. Devi, K. Devi and S. Sarasija, A novel approach for lung delivery of rifampicin-loaded liposomes in dry powder form for the treatment of tuberculosis, Lung India, 2015, 32(4), 331-338.

40 C. Rodrigues, G. Paula, M. Prietob and B. de Castro, Interaction of rifampicin and isoniazid with large unilamellar liposomes: spectroscopic location studies, Biochim. Biophys. Acta, 2003, 1620, 151-159.

41 P. Kadare, P. Maposa, A. Dube and C.C. Maponga, Encapsulation of isoniazid in chitosan-gum arabic and poly (lactic-co-glycolic acid) PVA particles to provide a sustained release formulation, J. Pharm. Pharmacol., 2014, S (1), 01-06.

42 I.M. El-Sherbiny, N.M. El-Baz and M.H. Yacoub, Inhaled nano- and microparticles for drug delivery, Glob. Cardiol. Sci. Pract., 2015, 2.

43 C. Kelly, C. Jefferies and S. Cryan, Targeted liposomal drug delivery to monocytes and macrophages, J. Drug Deliv., 2011, 11.

44 C. Singh, L.V.S.K. Koduri, A. Singh and S. Suresh, Novel potential for optimization of antitubercular therapy: pulmonary delivery of rifampicin lipospheres, Asian. J. Pharma. Sci., 2015, 10, 549-562.

45 J. Chuan, Y. Li, L. Yang, X. Sun, Q. Zhang, T. Gong and Z. Zhang, Enhanced rifampicin delivery to alveolar macrophages by solid lipid nanoparticles, J. Nanopart. Res., 2013, 15, 1634. 